RESEARCH ARTICLE

\title{
Schistosoma mansoni-Hepatitis B co-infection among adult patients with periportal fibrosis: a cross sectional study
}

\author{
Daniel W. Gunda ${ }^{1,2}$, Elizabeth F. Mtui ${ }^{1}$, Semvua B. Kilonzo ${ }^{1,2}$, Benson R. Kidenya ${ }^{3}$, Humphrey D. Mazigo \\ ${ }^{1}$ Department of Medicine, Catholic University of Health and Allied Sciences, Mwanza Tanzania \\ ${ }^{2}$ Department of Medicine, Bugando Medical Center, Mwanza Tanzania \\ ${ }^{3}$ Department of Biochemistry and Molecular Biology, Catholic University of Health and Allied Sciences, Mwanza \\ Tanzania \\ ${ }^{4}$ Department of Parasitology, Catholic University of Health and Allied Sciences, Mwanza Tanzania
}

\begin{abstract}
Objectives: Chronic Schistosoma mansoni infection is a common cause of periportal fibrosis in Sub Saharan Africa. About 20 million people are suffering complications of chronic $S$. mansoni infection with an annual mortality of 0.2 million people. The outcome of periportal fibrosis is highly modified by hepatitis $B$ co-infection which may cause a rapid progression to fibrosis and decompensation. In Tanzania both S. mansoni and hepatitis B are highly endemic; however, the co-infection among patients with periportal fibrosis in the hospital setting has not been described.

Methods: A cross-sectional study was done among patients with $S$. mansoni related periportal fibrosis at Bugando hospital. A minimum sample of 193 patients was calculated and, patients' clinical, laboratory, ultrasound and endoscopic data were analyzed using STATA 13. The prevalence of $S$. mansoni-hepatitis B co-infection was calculated and its correlates were determined by logistic regression model.
\end{abstract}

Results: In total 250 patients were analyzed in this study and, $40(16.0 \%)$ were found to have S. mansoni-Hepatitis B co-infection who were more likely to have higher AST levels, (58 vs. 38U/L; OR: 1.03; $p=0.033$ ), higher APRI levels, (1.8 vs. 1.05 ; OR: $2.1 ; P=0.03)$; ascites, (OR: $2.9 ; p=0.049$ ) with higher mortality, (OR: $2.9 ; p=0.032$ ).

Conclusions: The S. mansoni-Hepatitis B co-infection is common among patients with periportal fibrosis. The correlates found in this study, suggest that co-infected patients are more likely to have a severe liver injury with increased risk of severe fibrosis, decompensation, and mortality. Regular screening for hepatitis B and vaccination of people at-risk is highly suggested in this study. J Microbiol Infect Dis 2020; 10(3):136-143.

Keywords: Periportal fibrosis, Active S. mansoni, Hepatitis B co-infection, APRI score

\section{INTRODUCTION}

Schistosomiasis is a parasitic disease affecting more than 240 million people globally and it is of particular importance is Sub Saharan Africa (SSA). More than $90 \%$ of the global burden of schistosomiasis is concentrated in SSA where Schistosoma mansoni is the commonest [1]. About 54 million people are infected by $S$. mansoni and 400 million are at risk of infection. Most people are chronically infected and over 20 million are estimated to be suffering from complications of chronic $S$. mansoni infection with high mortality. Every year nearly 0.2 million loss of lives in Sub Saharan Africa is attributable to complications of chronic Schistosoma
mansoni infection $(1,2]$. Tanzania is the second most affected country in SSA where Schistosoma mansoni is highly endemic in Lake Zone part of the country $[3,4]$. In the lake zone part of Tanzania, high transmission of $S$. mansoni still occurs especially in communities that are engaged in freshwater activities like fishing $[1,5)]$ and most people are chronically infected. Community-based studies indicate that up to $42 \%$ of studied participants were found to have $S$. mansoni associated periportal fibrosis [6,7]. In the hospital setting, periportal fibrosis is a common cause of morbidity and mortality in the Lake Zone part of 
our country. for instance in 2014 Awilly et al. indicated that $70 \%$ of patients who were admitted due to hematemesis at Bugando had bleeding varices and about $60 \%$ of them had associated active $S$. mansoni infection and about $13(14.3 \%, \mathrm{n}=91)$ participants died within 2 months of diagnosis [8].

The outcome of people with periportal fibrosis is influenced by several factors including hepatitis co-infection. Literatures suggest that the coinfection of Hepatitis B virus (HBV) with $S$. mansoni may elongate the carriage state and it frequently proceeds to chronic hepatitis with extensive cirrhosis $[9,10)$, thus increasing the risk of morbidity from liver decompensation. Hepatitis B infection is highly prevalent in countries where S.mansoni is also highly endemic. Prior studies among patients with periportal fibrosis in Africa indicated that 16-33\% had S.mansoni-HBV co-infection [11,12]. In Tanzania, both HBV and $S$. mansoni are exceedingly common, however, published data regarding these two co-infections and its correlates are still scarce. This information is important in plans aiming at improving the outcome of this subgroup of patients in areas similar to our setting.

\section{METHODS}

This was a clinic-based cross-sectional study using retrospective data involving all adult patients with periportal fibrosis diagnosed between January 2015 and December 2018 at Bugando Medical Center (BMC). BMC is a university teaching hospital which is operating under super-specialized units since 2015. Gastroenterology and hepatology is one of the units under internal medicine with periportal fibrosis and cirrhosis being some of the common causes of specialist consultation.

The periportal fibrosis was diagnosed sonographically as done previously [13]. Details regarding portal vein and splenic diameters and presence or absence of ascites were documented. Diagnosis of active $S$. mansoni was based on positive Urine for Circulating Cathodic Antigen (CCA) or stool Kato Katz (KK). The diagnosis of HBV was based on a positive rapid test for HBsAg. Markers of liver injury i.e. Aspartate aminotransferase (AST) and Alanine aminotransferase (ALT), serum albumin and Full blood picture (FBP) were also done. All patients underwent upper digestive tract endoscopy to assess the presence of esophageal varices and were prescribed on biannual praziquantel (PZQ). Propranolol was added if patients had small varices and large varices were additionally band ligated.

A minimum sample size of 193 was estimated from two sample proportion formula, assuming hepatic decompensation will commonly lead to higher mortality of around $64 \%$ among Periportal Fibrosis patients co-infected with hepatitis $B$ with a morality difference of $15 \%$ [14]. A clinic registry was used to identify all patients who were diagnosed to have PPF at BMC during the study period. Registration numbers were noted and patients' files were retrieved. Sociodemographic data, clinical presentation like abdominal distension, hematemesis and melaena, Ultrasound (UTS) details including spleen diameter (SPD), portal vein diameter (PVD) and ascites; test results for $S$. mansoni, FBP, AST, ALT and serum Albumin, endoscopy results and survival status (alive or death) were retrieved for analysis.

Data were computerized using Epi data version 3.1 and STATA version 13 (Stata Corp LP, college station, TX) was used for analysis. Continuous variables were summarized as medians with interquartile range (IQR), while categorical variables were summarized as proportions with percentages. AST to platelet count (PTC) ratio index (APRI) was calculated as done previously [15]. S. mansoni- HBV coinfection was calculated and expressed as a percentage with 95\% Confidence Interval (Cl) and its correlates including markers of liver inflammation (AST, ALT), fibrosis (APRI values), decompensation (ascites, serum albumin) and death were assessed. The odds ratio (OR) with $95 \% \mathrm{Cl}$ was calculated by univariate followed by a multivariate logistic regression model to assess the degree of correlation between various factors and the outcome of interest. Factors with $p<0.2$ on the univariate model were subsequently included in the multivariate model. Factors were considered to have an independent correlation with the outcome of interest if the $p$-value was $<0.05$. The goodness of fit for the final logistic model was assessed by Hosmer-Lemeshow and the area under the receiver operating characteristics (ROC) curve, 
Hosmer-Lemeshow chi-square and p-value were reported.

\section{Ethical Clearance}

The permission to conduct and publish the findings from this study was sought from the catholic university of health and allied sciences (CUHAS)/BMC joint ethical committee with an ethical clearance certificate number 907/2019. The patients' information was handled by the researcher alone and their identifiers including names and registration numbers were not included in the final analysis to further maintain confidentiality.

\section{RESULTS}

\section{General Characteristics}

In this study, a total of 250 patients with periportal fibrosis were analyzed. The majority, $180(72.0 \%)$ were male participants with a median age of 41 (IQR: 33 to 51 ) years and most of them, i.e. $n=215(86.0 \%)$ were married. Patients commonly presented with abdominal distension, i.e. $\mathrm{n}=171$ (68.4\%) and less than a third, i.e. $n=79(31.6 \%)$ presented with overt upper gastrointestinal hemorrhage. The median Albumin, AST and ALT were 30.0 (IQR: 25.0 to 34.0 ) $\mathrm{g} / \mathrm{L}, 38$ (IQR: 26.0 to 55.0 ) U/L and 35 (IQR: 23 to 55) U/L respectively. On ultrasound, the median PVD and SPD were 1.5 (IQR: 1.4 to 1.7 ) and 17 (IQR: 15 to 18$) \mathrm{cm}$ respectively and $141(56 \%)$ patients had ascites. Endoscopically about 108 (43.2\%) participants had esophageal varices (Table 1).

Prevalence and correlates of hepatitis coinfection among 250 study participants

Of the 250 analyzed patients, about 222 of them (88.8\%; 95\% $\mathrm{Cl}$ : 84.2 to 92.4 ) were found to have a positive test for $S$. mansoni, whereas about 44 of them $(17.6 \%$; $95 \% \mathrm{Cl}$ : 13.1 to 22.9$)$ had positive HBVsAg test. Of all respondents about 40 (16.0\%; 95\% 11.6 to 21.1$)$, patients were co-infected with S.mansoni and HBV in this study (Figure 1). On multivariate analysis the patients with $S$. mansoni-HBV co-infection were more likely to have liver damage with higher median AST levels, (58U/L vs. 38U/L; OR: 1.03; $95 \% \mathrm{Cl}$ : 1.0 to $1.1 ; \mathrm{p}=0.033)$; an increased risk of liver fibrosis with higher median APRI score,
(1.8; vs. 1.05 ; OR: 2.1 ; $95 \% \mathrm{Cl}: 1.1$ to 3.9 ; $\mathrm{P}=0.03$ ); decompensation with ascites, (OR: 2.9; $95 \% \mathrm{Cl}: 1.1$ to $8.3 ; \mathrm{p}=0.049)$ with higher odds of mortality, (OR: 2.9; 95\%Cl: 1.4 to $7.8 ; p=0.032$ ). The distribution of other factors was not different statistically (Table 2) and the test for the goodness of fit of the final model did not demonstrate any gross lack of fit, (area under ROC curve: 0.8401; Hosmer-Lemeshow chisquare (8): $8.2 ; p=0.4138$ ) (Figure 2).

Table 1: Study characteristics among 250 participants with Schistosoma periportal fibrosis

\begin{tabular}{|c|c|}
\hline Variable & $\mathbf{N}$ (\% or median) \\
\hline \multicolumn{2}{|l|}{ Socio-demographic } \\
\hline Male gender & $180(72.0)$ \\
\hline Age (years) & $250(41,[33-51])$ \\
\hline Married & $215(86.0)$ \\
\hline Peasants & $197(78.8)$ \\
\hline Engaging in Fishing & $24(9.6)$ \\
\hline Lake contact report & $238(95.6)$ \\
\hline Alcohol use report & $123(49.2)$ \\
\hline \multicolumn{2}{|l|}{ Clinical presentation } \\
\hline Abdominal distension & $171(68.4)$ \\
\hline Upper GIT bleeding & $79(31.6)$ \\
\hline \multicolumn{2}{|l|}{ Laboratory results } \\
\hline Platelet count $\left(10^{3} / \mu \mathrm{L}\right)$ & 250 (88.5 [66-128]) \\
\hline Hemoglobin (g/dL) & $250(7.5$ [5.4-9.3]) \\
\hline Albumin (g/L) & $199(30.0$ [25.0-34.0]) \\
\hline ASAT (U/L) & $250(38[26.0-55.0])$ \\
\hline $\operatorname{ALAT}(\mathrm{U} / \mathrm{L})$ & $250(35[23-55])$ \\
\hline \multicolumn{2}{|l|}{ Ultrasound results } \\
\hline Portal vein diameter $(\mathrm{cm})$ & $250(1.5[1.4-1.7])$ \\
\hline Splenic diameter (cm) & $250(17[15-18])$ \\
\hline Ascites & $141(56.4)$ \\
\hline \multicolumn{2}{|l|}{ Endoscopy Results } \\
\hline $\begin{array}{l}\text { Esophageal varices } \\
\text { present }\end{array}$ & $108(43.2)$ \\
\hline No esophageal varices & $142(56.8)$ \\
\hline
\end{tabular}




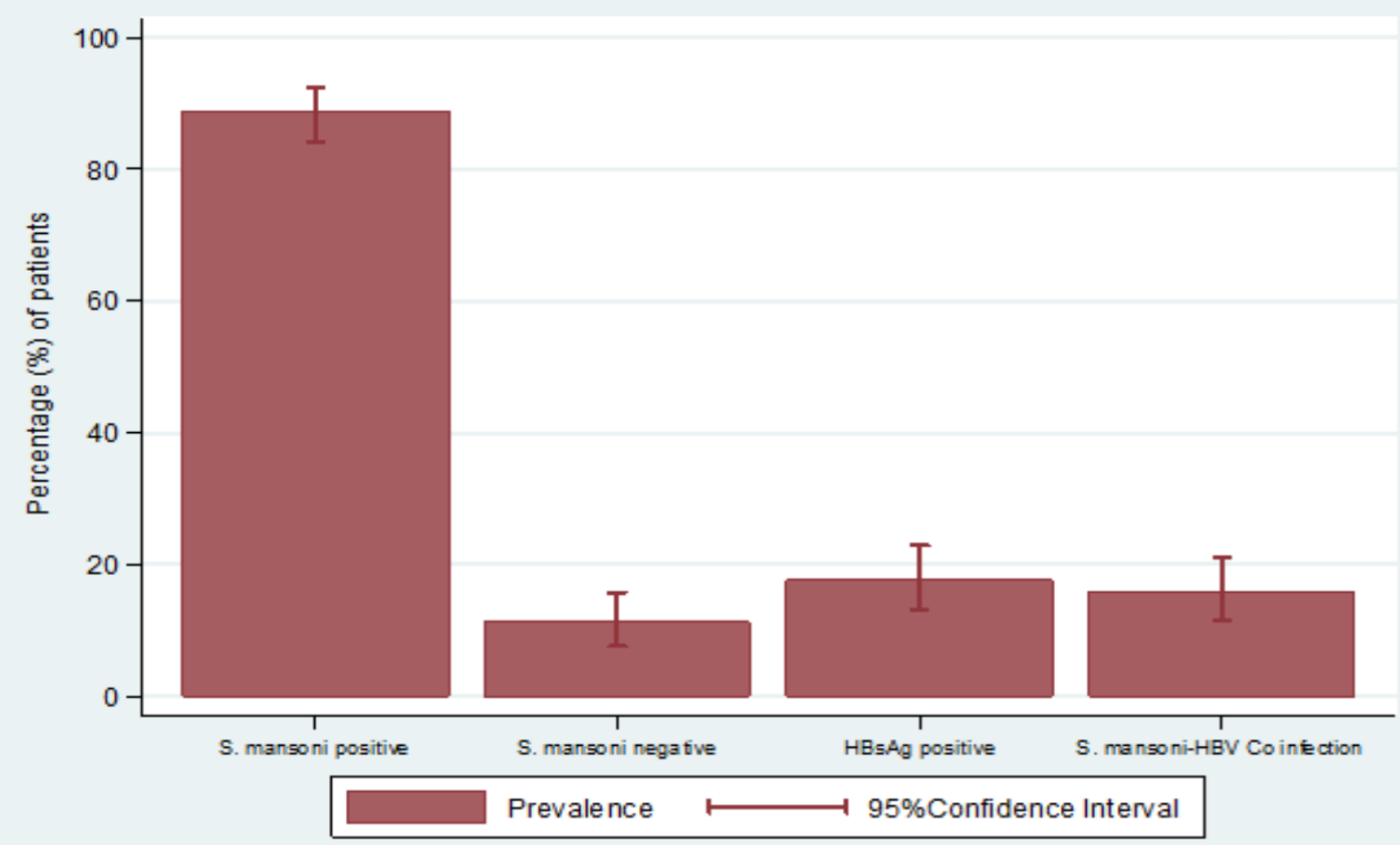

Figure 1: Distribution of Schistoma mansoni and HBV test results among 250 participatns

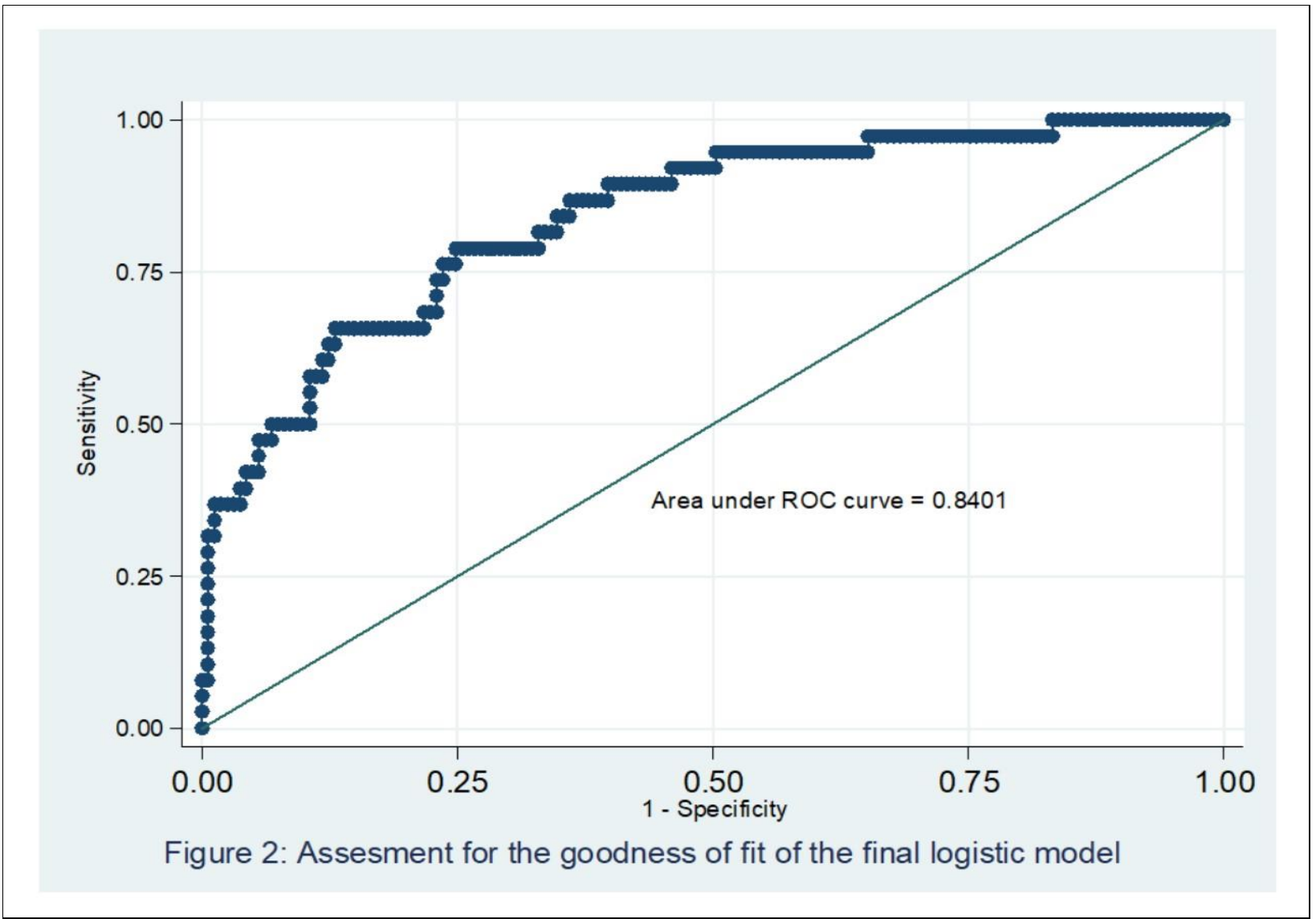


Table 2. Correlates of S. mansoni- HBV co-infection among 250 patients with periportal fibrosis.

\begin{tabular}{|c|c|c|c|c|c|c|}
\hline \multirow[t]{2}{*}{ Variables } & \multicolumn{2}{|c|}{ Schistosoma-HBV co-infection } & \multicolumn{2}{|c|}{ Un adjusted } & \multicolumn{2}{|c|}{ Adjusted } \\
\hline & No $(n=210)$ & Yes $(n=40)$ & OR $(95 \% \mathrm{Cl})$ & $\mathrm{p}$-value & OR $(95 \% \mathrm{Cl})$ & $p$-value \\
\hline Gender (Male) & $149(70.9)$ & $31(77.5)$ & $1.4(0.6-3.1)$ & 0.400 & & \\
\hline Age, (years) & $41[34-51]$ & 37 [32-53] & $1.0(0.9-1.01)$ & 0.683 & & \\
\hline Married & $27(12.9)$ & $8(20.0)$ & $1.0(0.2-1.4)$ & 0.237 & & \\
\hline Taking alcohol & $99(47.1)$ & $24(60.0)$ & $1.7(0.8-3.3)$ & 0.139 & $1.3(0.6-3.2)$ & 0.488 \\
\hline Engaging in Fishing & $21(10.0)$ & $3(7.5)$ & $0.7(0.2-2.5)$ & 0.624 & & \\
\hline UGIT bleeding & $52(24.8)$ & $12(30.0)$ & $1.3(0.6-2.7)$ & 0.487 & & \\
\hline SPD (cm) & $17(15-18)$ & $17(16-18)$ & $1.1(0.9-1.2)$ & 0.341 & & \\
\hline PVD (cm) & $1.5[1.4-1.7]$ & $1.51[1.2-1.6]$ & $0.5(0.2-1.3)$ & 0.260 & & \\
\hline \multicolumn{7}{|l|}{ Liver injury/fibrosis } \\
\hline ASAT(U/L) & 38 [24-48] & 58 [40-116] & $1.02(1.01-1.03)$ & $<0.001$ & $1.03(1.0-1.1)$ & 0.033 \\
\hline $\operatorname{ALAT}(\mathrm{U} / \mathrm{L})$ & $33.5[21-52]$ & $49.5[34-77]$ & $1.01(1.0-1.02)$ & 0.001 & $0.98(0.9-1.0)$ & 0.084 \\
\hline APRI score & $1.05[0.6-1.6]$ & $1.8[1.3-2.7]$ & $3.5(2.1-5.5)$ & $<0.001$ & $2.1(1.1-3.9)$ & 0.030 \\
\hline Ascites & $107(50.9)$ & $34(85.0)$ & $5.5(2.2-13.5)$ & $<0.001$ & $2.9(1.1-8.3)$ & 0.049 \\
\hline Albumin ( $g / L)$ & $30.1[27.8-41.5]$ & 30 [24-33] & $1.04(1.0-1.1)$ & 0.058 & $1.03(1.0-1.1)$ & 0.218 \\
\hline Esophageal Varices & $90(42.9)$ & $19(47.5)$ & $1.2(0.6-2.3)$ & 0.588 & & \\
\hline Outcome (Died) & $26(12.4)$ & $12(30.0)$ & $3.0(1.3-6.6)$ & 0.006 & $2.9(1.4-7.8)$ & 0.032 \\
\hline
\end{tabular}

\section{DISCUSSION}

The objective of this study was to determine the prevalence and correlates of Schistosoma mansoni-Hepatitis B co-infection among patients diagnosed with periportal fibrosis. In total, 40 $(16.0 \%)$ patients had S. mansoni- Hepatitis B co-infection in this study. The patients who had S. mansoni- Hepatitis B co-infection was more likely to have higher serum AST levels, higher APRI scores, and ascites with increased risk of mortality.

The prevalence of $S$. mansoni-Hepatitis B coinfection in this study is similar to a prevalence of $15.8 \%$ reported by Aquino et al. from Brazil [16), $16.1 \%$ reported in another study by Berhe et al. in Ethiopia [17) and prevalence rate of $19.6 \%$ reported earlier in 1991 by El-Sayed et al. from Egypt [18). However, the prevalence of $S$. mansoni-Hepatitis $\mathrm{B}$ co-infection in the current study is lower than the prevalence rate of $25.4 \%$ reported in a recent study from rural part of China [19); 33.0\% that was reported earlier in 1997 in a study from Egypt [12) and 58.4\% reported in another study by $\mathrm{Du}$ et al. from China in 2013 (20). On the contrary, the prevalence in the current study is much higher as compared to the prevalence of $5.3 \%$ reported among patients with PPF earlier in Ethiopia [11).

Several factors were assessed for their independent correlation with $S$. mansoniHepatitis B co-infection in this study. Patients with S. mansoni-Hepatitis B co-infection were more likely to have elevated serum levels of markers of liver injury and higher APRI fibrosis score. These findings are consistent with the previous findings in a study done by Andrade et al. [21) and another study from Egypt [22). These findings suggest that the $S$. mansoniHepatitis B co-infection increases the risk of 
much severe liver inflammation, damage, and subsequent severe hepatic fibrosis.

Aquino et al. in their study, indicated that patients with $S$. mansoni related periportal fibrosis and hepatitis B co-infection were more likely to have decompensated liver disease which frequently presented with ascites, low serum albumin and coagulopathy [23). Though coagulopathy wasn't assessed in this study, we similarly observed that patients who had $S$. mansoni-Hepatitis B co-infection were 2.9 times more likely to have ascites, with a tendency to towards having non-significant lower serum albumin levels (OR: $1.03, p=0.218$ ) as compared to the mono-infected counterparts.

While patients with S. mansoni-hepatitis B coinfection were more likely to be males in gender and taking alcohol in another study [21), this wasn't the case in our study where gender, alcohol and portal vein diameter had only a nonsignificant trend of association. A similar observation was also reported by Chisenga et al. among HIV patients who had both $S$. mansoni and hepatitis B co-infection. In this study, the $S$. mansoni-hepatitis B co-infection had non-significant trends with male gender but also a non-significant trend towards having a large portal vein in their study participants [24).

The odds of mortality in the current study were about 3 times higher among those who had $S$. mansoni-Hepatitis B co-infection as compared to mono-infected counterparts. Mortality has been reported previously to be considerably higher in this subgroup of patients similar to our findings [25). Bassily et al. indicated that the mortality was significantly higher among $S$. mansonihepatitis B co-infected patients $(64.0 \%$ vs. $22 \%)$ among those who were mono-infected [14) as compared to those who were mono-infected similar to our current findings ( $30.0 \%$ vs. $12.4 \%$, AOR: $2.9 ; p=0.032$ ).

These findings suggest that the $S$. mansoni and Hepatitis B co-infection is prevalent in this Schistosoma endemic area. The co-infection among patients with periportal fibrosis is rather a serious clinical entity that is associated with increased risk of severe liver damage, fibrosis, decompensation, and mortality. On clinical grounds, this presents a very challenging situation where both the prevention and treatment of the co-infection is difficult still in our setting [26). The available treatment modalities for HBV with a positive effect among S. mansoni co-infected patients like Entecavir and Pegylated
IFN $[27,28)$ are still extremely expensive as potential treatment options in our setting.

Otherwise regular screening for the co-infections is highly suggested in this study. These patients should be on regular PZQ doses as pre-primary prevention as $P Z Q$ has been shown to reverse the PPF significantly $[29,30)$. Vaccination against HBV in these patients should highly be considered. Based on a review in Tanzania, HBV seroprevalence is lowest among children most likely due to the wide coverage of hepatitis $B$ vaccination as per the expanded program of immunization in the country. Adult blood donors have the highest HBV seroprevalence of 10$21 \%$, and this emphasizes a call for universal screening and vaccination against HBV in all adult populations in the country [31).

This study is liable to several limitations. It is a single-center study thus its findings may not be generalizable. Being retrospective some information couldn't be retrieved including severity grading of liver fibrosis, bleeding indices and hepatitis panels. Otherwise, this is the first study to describe Schistosoma mansoni and hepatitis B co-infection in hospital-based patients in Tanzania and thus the results are highly important in devising strategies to improve the future outcome of these patients.

\section{ACKNOWLEDGMENTS}

Ethical clearance was obtained with certificate no $907 / 2019$

Authors' contributions: DWG, EFM, HDM\& SBK: participated in the conception, designing of the study and acquiring the data; DWG \& BRK did data analysis and interpretation; DWG: did manuscript drafting. All the authors critically reviewed the manuscript for its intellectual content and approved the final version.

Declaration of Conflicting Interests: The authors declare that they have no conflict of interest.

\section{Funding: Not applicable}

The authors would like to acknowledge the great support given by the department of medical records at BMC during retrieval of patients' information.

\section{REFERENCES}

1. WHO. Schistosomiasis: Progress Report 20012011 and Strategic plan 2012-2020. 2013:74.

2. Hotez PJ, Asojo OA, Adesina AM. Nigeria: "Ground Zero" for the High Prevalence Neglected Tropical Diseases. Public Library of Science; 2012. 
3. Brooker S, Kabatereine NB, Smith JL, Mupfasoni $\mathrm{D}$, Mwanje MT, Ndayishimiye O, et al. An updated atlas of human helminth infections: the example of East Africa. Int J Health Geogr 2009; 8:42.

4. Steinmann P, Keiser J, Bos R, Tanner M, Utzinger J. Schistosomiasis and water resources development: a systematic review, meta-analysis, and estimates of people at risk. Lancet Infect Dis 2006; 6(7):411-425.

5. Hotez PJ, Asojo OA, Adesina AM. Nigeria: "Ground Zero" for the high prevalence of neglected tropical diseases. PLoS Negl Trop Dis 2012; 6(7):e1600.

6. Kardorff R, Gabone RM, Mugashe C, Obiga D, Ramarokoto CE, Mahlert C, et al. Schistosoma mansoni-related morbidity on Ukerewe Island, Tanzania: clinical, ultrasonographical and biochemical parameters. Trop Med Int Health 1997; 2(3):230-239.

7. Mazigo HD, Nuwaha F, Dunne DW, Kaatano GM, Angelo T, Kepha S, et al. Schistosoma mansoni Infection and Its Related Morbidity among Adults Living in Selected Villages of Mara Region, NorthWestern Tanzania: A Cross-Sectional Exploratory Study. Korean J Parasitol 2017; 55(5):533-540.

8. Chofle AA, Jaka H, Koy M, Smart LR, Kabangila $R$, Ewings $F M$, et al. Oesophageal varices, schistosomiasis, and mortality among patients admitted with haematemesis in Mwanza, Tanzania: a prospective cohort study. BMC Infect Dis 2014;14(1):303.

9. Abruzzi A, Fried B, Alikhan SB. Coinfection of Schistosoma species with hepatitis b or hepatitis C viruses. Advances in Parasitology: Elsevier; 2016. p. 111-231.

10. Conceição MJ, Argento CA, Chagas VLA, Takiya CM, Moura DC, Silva SC. Prognosis of schistosomiasis mansoni patients infected with the hepatitis B virus. Memórias do Instituto Oswaldo Cruz 1998; 93:255-8.

11. Berhe N, Myrvang B, Gundersen SG. The intensity of Schistosoma mansoni, hepatitis B, age, and sex predict levels of hepatic periportal thickening/fibrosis (PPT/F): a large-scale community-based study in Ethiopia. The American J Tropic Med Hygien 2007; 77(6):1079-1086.

12. El-Sayed H, Abaza S, Mehanna S, Winch PJ. The prevalence of hepatitis $B$ and $C$ infections among immigrants to a newly reclaimed area endemic for Schistosoma mansoni in Sinai, Egypt. Acta Tropic 1997; 68(2):229-37.

13. Richter J, Hatz C, Campagne G, Bergquist N, Jenkins JM. Ultrasound in schistosomiasis: a practical guide to the standard use of ultrasonography for assessment of schistosomiasis-related morbidity: Second international workshop, October 22-26 1996, Niamey, Niger: Geneva: World Health Organization2000.
14. Bassily S, Farid Z, Higashi GI, Kamel IA, El-Masry $\mathrm{NA}$, Watten $\mathrm{RH}$. Chronic hepatitis $B$ antigenemia in patients with hepatosplenic schistosomiasis. J Trop Med Hyg 1979; 82(11-12):248-251.

15. Chou R, Wasson N. Blood tests to diagnose fibrosis or cirrhosis in patients with chronic hepatitis $C$ virus infection: a systematic review. Ann Intern Med 2013; 158(11):807-820.

16. Aquino RT, Chieffi PP, Catunda SM, et al. Hepatitis $B$ and $C$ virus markers among patients with hepatosplenic mansonic schistosomiasis. Rev Inst Med Trop Sao Paulo 2000; 42(6):313-20.

17. Berhe N, Myrvang B, Gundersen SG. The intensity of Schistosoma mansoni, hepatitis B, age, and sex predict levels of hepatic periportal thickening/fibrosis (PPT/F): a large-scale community-based study in Ethiopia. Am J Trop Med Hyg 2007; 77(6):1079-1086.

18. el-Sayed HF, Abaza SM, Mehanna S, Winch PJ. The prevalence of hepatitis $B$ and $C$ infections among immigrants to a newly reclaimed area endemic for Schistosoma mansoni in Sinai, Egypt. Acta Trop 1997; 68(2):229-237.

19. Zhang Y, Xie Y, Chen Q, Chen X, Dong Z, Tan X. Prevalence and co-infection of schistosomiasis/hepatitis B among rural populations in endemic areas in Hubei, China. Trans R Soc Trop Med Hyg 2020;114(3):155-161.

20. Du H. [Relationship between advanced schistosomiasis and HBV infection]. Zhongguo Xue Xi Chong Bing Fang Zhi Za Zhi 2013; 25(1):115, 7.

21. Andrade J, Silva LD, Guimarães CM, BassettiSoares E, Cambraia RD, Couto O, et al. Chronic hepatitis $B$ and liver schistosomiasis: a deleterious association. Transactions of the Royal Society of Tropical Medicine and Hygiene 2014;108(3):159164.

22. Omar $\mathrm{HH}$, Taha SA, Hassan WH, Omar $\mathrm{HH}$ Impact of schistosomiasis on the increased incidence of occult hepatitis B in chronic hepatitis C patients in Egypt. J Infect Public Health 2017; 10(6):761-765.

23. Aquino RTRD, Chieffi PP, Catunda SDM, et al. Hepatitis $B$ and $C$ virus markers among patients with hepatosplenic mansonic schistosomiasis. Revista do Instituto de Medicina Tropical de Sao Paulo 2000; 42(6):313-320.

24. Chisenga C, Sinkala E, Chilengi $R$, et al. Prevalence and Clinical Significance of Schistosomiasis-Chronic Hepatit B Virus CoInfection in Zambia. BMJ Global Health. 2017; 2(Suppl 2): A8-A9.

25. Madwar MA, Shaker MK, Atta MA, El Khashaab $\mathrm{TH}$, Mohamed MK. A prospective study: prediction of the first variceal hemorrhage in schistosomal 
and non-schistosomal liver disease. J Egypt Public Health Assoc 1997; 72(3-4):395-409.

26. Gasim GI, Bella A, Adam I. Schistosomiasis, hepatitis $B$ and hepatitis $C$ co-infection. Virology $J$ 2015;12(1):19.

27. Draz HM, Mahmoud SS, Ashour E, Shaker YM, Wu CH, Wu GY. Effects of PEG-interferon-alpha$2 \mathrm{~A}$ on Schistosoma mansoni infection in mice. Journal of Parasitology. 2010;96(4):703-708.

28. Huang L-H, Qiu Y-W, Hua H-Y, Niu X-H, Wu P-F, $\mathrm{Wu} \mathrm{H}-\mathrm{Y}$, et al. The efficacy and safety of entecavir in patients with advanced schistosomiasis coinfected with hepatitis B virus. International Journal of Infectious Diseases. 2013; 17(8):e606e9.

29. El-Lakkany NM, Hammam OA, El-Maadawy WH, Badawy AA, Ain-Shoka AA, Ebeid FA. Antiinflammatory/anti-fibrotic effects of the hepatoprotective silymarin and the schistosomicide praziquantel against Schistosoma mansoni-induced liver fibrosis. Parasites \& vectors. 2012;5(1):9.

30. Martins-Leite P, Gazzinelli G, Alves-Oliveira L, et al. Effect of chemotherapy with praziquantel on the production of cytokines and morbidity associated with schistosomiasis mansoni. Antimicrobial agents and chemotherapy. 2008;52(8):2780-6.

31. Kilonzo SB, Gunda DW, Mpondo BC, Bakshi FA, Jaka $H$. Hepatitis B virus infection in Tanzania: current status and challenges. Journal of tropical medicine. 2018;2018. 